\title{
SERVIÇO DE ATENDIMENTO FARMACÊUTICO AO IDOSO: relato de experiência de educação em saúde
}

\author{
PHARMACIST CUSTOMER SERVICE ELDERLY: \\ report of health education experience
}

\section{Sandna Larissa Freitas dos Santos', Rainne de Oliveira Almeida', Carlos Eduardo Quirino Paiva', Karla Bruna Nogueira Torres Barros², Maria Luísa Bezerra de Macedo Arraes²}

\section{RESUMO}

O âmbito de educação em saúde ao idoso, torna-se uma maneira de ampliar o debate acerca da saúde, bem como de favorecer a formação de profissionais com competência para cuidar nesse cenário, promovendo o desenvolvimento do conhecimento e com isso melhorar a qualidade de vida e saúde das pessoas envolvidas neste processo. Objetivo: Apresentar um relato de experiências de ações educativas em saúde á população idosa da casa de acolhida Remanso da Paz,- Quixadá-CE, por meio de um projeto de extensão SAFI - Serviço de Atendimento Farmacêutico ao paciente idoso, desenvolvido pelos acadêmicos do curso de Farmácia da Faculdade Católica Rainha do Sertão, Quixadá-CE, realizado no período de agosto a dezembro de 2015. Relato da Experiência: Foi baseado em orientação e aconselhamento sobre o uso adequado de medicamentos, desenvolvendo atividades de educação em saúde, produzindo palestras informativas sobre as doenças crônicas que mais acometem a população idosa, além da execução de teste de glicemia e verificação de pressão arterial para averiguação da terapia medicamentosa. Dentre os temas abordados destacam: uso racional de medicamentos, uso de preparações caseiras de plantas medicinais, diabetes e hipertensão, problemas reumáticos, doenças cardiovasculares, sedentarismo/ alimentação, e doenças respiratórias. Por fim, foi disponibilizado material educativo sobre orientação de ações de qualidade de vida ao idoso e a importância da atuação do Farmacêutico na saúde desta população. Conclusão: Dessa forma, no contexto de educação em saúde se sobrepõe o conceito de promoção da saúde, como uma definição mais ampla de um processo que abrange a participação de toda a população no contexto de sua vida cotidiana do idoso e não apenas das pessoas sob risco de adoecer, evidenciando que as ações desenvolvidas são essenciais na melhoria da qualidade de vida do idoso.

Descritores: Atenção Farmacêutica; Saúde do Idoso; Educação em Saúde.

\begin{abstract}
The health education under the elderly becomes a way to broaden the debate about health and to promote the training of professionals responsible for care in this scenario, promoting the development of knowledge and thus improve the quality of life and health of the people involved in this process. Objective: To present an account of experiences of educational actions in health elderly population will welcome the home of Remanso Paz Quixadá-CE, through an extension project SAFI - Pharmaceutical Service to elderly patient, developed by students of the course pharmacy Catholic School Queen of Hinterland, Quixadá-CE, conducted from August to December 2015. Report of Experience: it was based on guidance and advice on the proper use of drugs, developing health education activities, producing informative talks on chronic diseases that most affect the elderly, as well as blood glucose test run and checking blood pressure for investigation of drug therapy. Among the topics highlighted: rational use of drugs, use of homemade herbal preparations, diabetes and hypertension, rheumatic problems, cardiovascular disease, sedentary lifestyle / nutrition and respiratory diseases. Finally, educational material on guidance quality of life actions to the elderly and the importance of the pharmacist involved in the health of this population was made available. Conclusion: Thus, in health education context overlaps the concept of health promotion, such as a broader definition of a process that includes the participation of the entire population in the context of their everyday life of the elderly and not only people under risk of becoming ill, showing that the developed actions are essential to improve the quality of life of the elderly.
\end{abstract}

${ }^{1}$ Acadêmica(o) de Farmácia na Faculdade Católica Rainha do Sertão (FCRS), Quixadá, CE, Brasil.

${ }^{2}$ Graduada em Farmácia pela Faculdade Católica Rainha do Sertão (FCRS), Quixadá, CE, Brasil. 


\section{Introdução}

A proporção de idosos na população brasileira vem crescendo rapidamente, configurando um dos maiores desafios da saúde pública contemporânea, ou seja, a queda das taxas de fecundidade e o aumento da expectativa de vida começaram a alterar sua estrutura, estreitando progressivamente a base da pirâmide populacional e alargando seu ápice. Isto o que oocasiona redefinição nas responsabilidades familiares e nas demandas por políticas públicas de saúde, alterando até mesmo as relações de gênero no seio familiar ${ }^{1}$.

Em geral, com o aumento da idade cronológica, ocorre uma maior prevalência de condições crônicas de saúde, o que predispõe os idosos um maior consumo de medicamentos, apresentando assim, peculiaridades se comparado ao restante da população. Soma-se, assim com a falta de qualidade da terapia medicamentosa, a presença de polifarmácia, o uso de medicamentos inadequados e a duplicidade terapêutica, o que contribui para ocorrência de reações adversas e interações medicamentosas².

O conhecimento do perfil de utilização de medicamentos da população geriátrica é essencial relacionado ao delineamento de estratégias de prescrição racional de medicamentos para idosos dependentes do setor público e privado $^{3}$. Estudos têm mostrado que a intervenção farmacêutica por meio de ações educativas e orientações sobre 0 regime terapêutico traz benefícios à saúde do paciente e ao processo de promoção da saúde. Essa orientação deve ser destinada não só ao paciente idoso, mas também ao seu acompanhante, familiar, cuidador e, ainda, ao médico prescritor e demais profissionais de saúde envolvidos diretamente na assistência à saúde ${ }^{2-3}$.

É essencial realizar habilidades de comunicação com os pacientes em Atenção Farmacêutica para alcançar melhores resultados com as intervenções propostas ${ }^{4}$. Entretanto, 0 aconselhamento acerca do uso racional de medicamento é prática importante para a população em geral e em especial para o idoso, em função da presença frequente de múltiplas patologias, requerendo terapias diferentes, as quais podem resultar no uso concomitante de vários medicamentos².

Diante disso , o estudo tem como objetivo apresentar um relato de experiência de ações educativas e orientações sobre o regime terapêutico como aspecto benéfico à saúde do paciente idoso e ao processo de promoção da saúde, apresentando estratégias facilitadoras para implantar o serviço de Atenção Farmacêutica ao idoso e que esses recursos sejam um referencial aos estabelecimentos de saúde.

\subsection{A POLIMEDICAÇÃO EM IDOSOS}

Nos últimos anos a perspectiva de vida tem crescido numericamente e com isso a quantidade de idosos também, aumentando o índice populacional e a procura por medicamentos para auxiliar em uma melhor qualidade de vida. Isso se deve á ações de saúde pública como vacinação, saneamento, programas de prevenção e promoção da saúde do idoso e também, aos avanços técnico - científicos das ciências do âmbito da saúde ${ }^{5}$.

O fácil acesso aos medicamentos, características do médico e do paciente, bem como a reduzida frequência de uso de meios não-farmacológicos para as doenças, atuam como os diversos fatores que levam à polifarmácia. Porém, evitar a polifarmácia é de importância significativa, pois quanto maior o número de medicamentos utilizados, maior é a chance de interações medicamentosas, salientando-se que nas fases de testes de ensaios clínicos feitos com fármacos a serem comercializados, a população idosa é muitas vezes excluída (por questões éticas, entre outras razões), de modo que as informações sobre a eficácia e a segurança do uso nesse grupo acabam sendo escassas ${ }^{6}$.

A prática da polifarmácia em muitos casos se justifica pelo quadro de doenças apresentado pelo indivíduo, fornecendo benefício no controle de muitas condições crônicas, e tem sido definida de diferentes formas desde a qualidade até a quantidade de medicamentos utilizados. A definição qualitativa é a prescrição, administração ou uso de mais medicamentos do que está clinicamente indicado ao paciente. Já a definição quantitativa da polifarmácia apresenta várias classificações, partindo desde o uso de dois ou mais medicamentos, até o uso de seis ou mais ${ }^{7}$.

Para se entender e prevenir esta prática, além de tratar suas complicações, o profissional farmacêutico que atende o público geriátrico deve ter o conhecimento que abrange desde as alterações orgânicas fisiológicas do envelhecimento, que irá influenciar no metabolismo dos fármacos, bem como a farmacologia das substâncias prescritas, suas possíveis interações medicamentosas e efeitos adversos. Deve estar ciente também das dificuldades encontradas pelo idoso em orientar-se nas prescrições médicas e na dificuldade do apego terapêutico, e na realidade socioeconômica individual desses pacientes, sendo que este é um fator muito importante na saúde coletivå․ 


\subsection{A IMPORTÂNCIA DA ATENÇÃO FARMACÊUTICA AO IDOSO}

O termo Atenção Farmacêutica foi adotado e oficializado no Brasil a partir de deliberações entre a Organização Pan-Americana de Saúde (OPAS) em 2002, Organização Mundial da Saúde (OMS ), Ministério da Saúde (MS), entre outros. Definida como: "um modelo de prática farmacêutica, unindo atitudes, valores éticos, comportamentos, habilidades, compromissos e co-responsabilidades na prevenção de doenças, promoção e recuperação da saúde, de forma integrada à equipe de saúde. É a interação direta do farmacêutico com o usuário, visando uma farmacoterapia racional e a obtenção de resultados definidos e mensuráveis, voltados para a melhoria da qualidade de vida ${ }^{9}$.

As atribuições do farmacêutico no âmbito da atenção à saúde estão associadas em obter e manter dados sobre os medicamentos utilizados pelo paciente e informações relevantes sobre sua saúde. Quando inexistentes, identificar problemas relativos aos medicamentos, efeitos colaterais, interações medicamentosas, uso incorreto de medicamentos, além de elaborar e implementar o plano de atenção farmacêutica (educação, orientação, intervenções e parâmetros farmacêuticos) $)^{4}$. Quando executadas, em sua totalidade ou em parte, essas ações acrescentarão valor à terapia medicamentosa por contribuírem positivamente para o uso seguro e custo-efetivo dos medicamentos, levando a resultados positivos e aprimorando a atenção à saúde ${ }^{5}$.

Porém, de acordo com Gomes e Caldas, $(2008)^{10}$ faz-se necessário mediante o conhecimento da realidade dos idosos do Brasil, bem como, observando-se as dificuldades e necessidades dessa população em relação ao estado favorável de saúde, estabelecer com esse paciente idoso uma propedêutica onde se considere o uso racional de medicamentos. Por isso, é preciso repensar a atuação dos profissionais de saúde no âmbito de sua qualificação para lidar com o idoso.

\subsection{EDUCAÇÃO EM SAÚDE DO IDOSO}

A educação em saúde é aquela que se baseia no diálogo e na troca de saberes, constituindo-se um verdadeiro intercâmbio entre o saber científico e o popular, de modo que cada um tem muito a ensinar e muito a aprender. A comunicação na atenção à saúde é algo que se constrói em uma ação intencional, dirigida e orientada para um interesse concreto. Os processos comunicativos baseiam-se em escutar o outro para compreender quais as suas crenças, sua situação e suas possibilidades e poder atuar conjuntamente ${ }^{11}$.

A educação, portanto, é um dos meios para vencer os desafios impostos aos idosos pela idade e pela sociedade, propiciando-Ihes o aprendizado. Como exemplo pode-se citar que programas de educação em osteoporose, sem dúvida, aumentam o nível de conhecimento, atenção e cuidados com a saúde óssea, mostrando a necessidade de contínuo esforço em projetos dessa natureza ${ }^{12}$.

No processo de envelhecimento, o idoso sofre modificações biológicas, fisiológicas, cognitivas, patológicas e socioeconômicas necessitando, portanto, de atenção especial. No entanto, as particularidades da idade não podem definir que o idoso seja um ser doente , e sim que tais modificações podem ser adaptáveis a uma vida ativa e saudável. Torna-se fundamental que os profissionais de saúde tomem consciência dos fatores determinantes desse processo, compreendendo sua complexidade e atuando em prol da promoção da saúde desses idosos. Com isso, uma das alternativas mais importantes para assegurar a autonomia e independência do idoso, como também o envelhecer saudável, são as ações educativas ${ }^{13}$.

A educação em saúde então visa promover o desenvolvimento do conhecimento e com isso melhorar a qualidade de vida e saúde das pessoas envolvidas neste processo. A implementação de atividades que envolvam ações educativas com idosos, torna-se uma maneira de ampliar o debate acerca dessas questões, bem como de favorecer a formação de profissionais com competência para cuidar nesse cenário ${ }^{14}$.

\section{Relato de Experiência}

O Serviço de Atendimento Farmacêutico ao Idoso (SAFI) desenvolveu-se na casa de apoio aos idosos "Remanso da Paz" município de Quixadá-CE, a qual é uma instituição filantrópica que realiza atividades ocupacionais e educativas, sendo mediadas por profissionais voluntários. A experiência foi desenvolvida por acadêmicos do curso de Farmácia da Faculdade Católica Rainha do Sertão-FCRS- Quixadá-CE, com o intuito de aperfeiçoar o conhecimento nas atividades 
relacionadas à Atenção Farmacêutica, com ênfase ao paciente idoso, com a participação de 22 idosos, elaborado no período de agosto a dezembro de 2015.

Destes , $16(72,7 \%)$ eram mulheres e $6(27,3 \%)$ homens, na faixa etária de 60 a 90 anos. Quanto ao estado civil $12(54,54 \%)$ eram viúvos, $4(18,18 \%)$ solteiros, $4(18,18 \%)$ casados e $2(9,10 \%)$ separados. Sobre a escolaridade, 9 $(40,90 \%)$ possuem ensino médio, 6 (27,3\%) ensino fundamental, $6(27,3 \%)$ não são alfabetizados e $1(4,5 \%)$ ensino superior. Com relação as comorbidades, houve maior prevalência entre os participantes de hipertensão (58\%) e diabetes (42\%). Para a coleta desses dados foi aplicado um questionário.

Esses dados assemelham-se com os de Leite-Cavalcanti ${ }^{1}$ et al. (2009) ${ }^{15}$ o qual em uma amostra de 117 idosos, 78,6 \% utilizam medicamentos e 82,1 \% possuíam alguma doença crônica não-transmissível, sendo mais frequentes a hipertensão arterial (56,4\%), as dislipidemias (33,3\%) e o diabetes mellitus $(20,5 \%)$. Santos et al. $(2013)^{16}$, em sua pesquisa evidenciou o sexo feminino com maior prevalência de hipertensão, sendo que $70 \%$ de internações por idosos foram causadas por Diabetes mellitus e $65 \%$ por Hipertensão.

O projeto contou com a realização de acompanhamento farmacoterapêutico (Figura 01) aos pacientes portadores das doenças crônicas, prestando orientação e aconselhamento sobre o uso adequado de medicamentos, bem como avaliação da terapia medicamentosa, desenvolvendo atividades de educação em saúde voltadas para a melhoria da qualidade de vida de pacientes idosos, e assim intensificando o processo ensino e aprendizagem, incluindo o discente na prática de Atenção e Farmacêutica na geriatria.

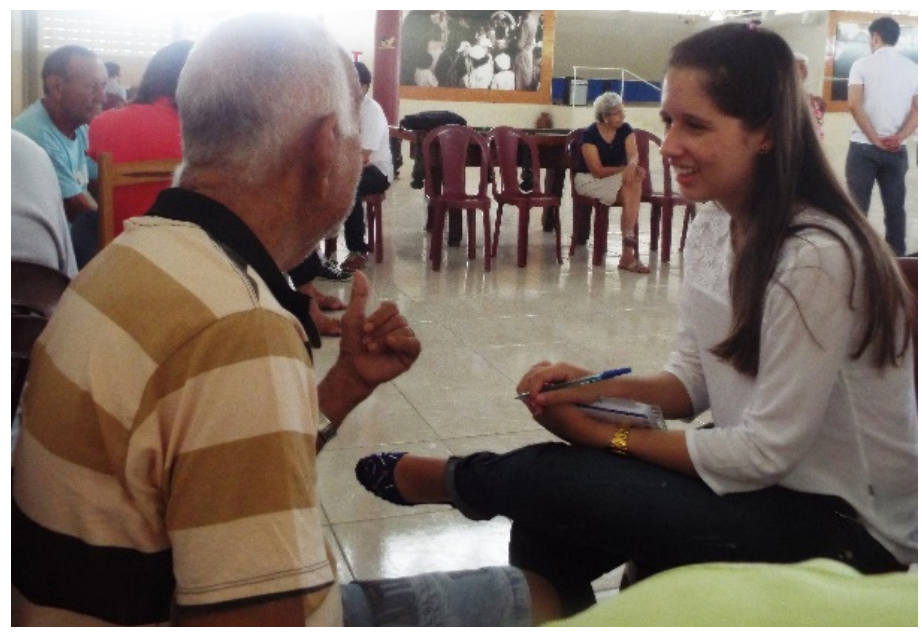

Figura 01 - Conversa com idoso para acompanhamento farmacoterapêutico.

Os encontros ocorriam semanalmente, por meio de palestras e rodas de conversas explicativas (Figura 02 e 03), com uso de cartazes explanando as causas e a importância do controle das diversas patologias, e esclarecimento das dúvidas dos idosos participantes. Foram abordados temas como, uso de plantas medicinais, com aconselhamento de como usar corretamente e as preparações caseiras, alimentação saudável, uso racional de medicamentos, e doenças de maior ocorrência na população idosa, como Hipertensão, Diabetes, Osteoporose, Acidente Vascular Encefálico, dentre outras ${ }^{3}$.

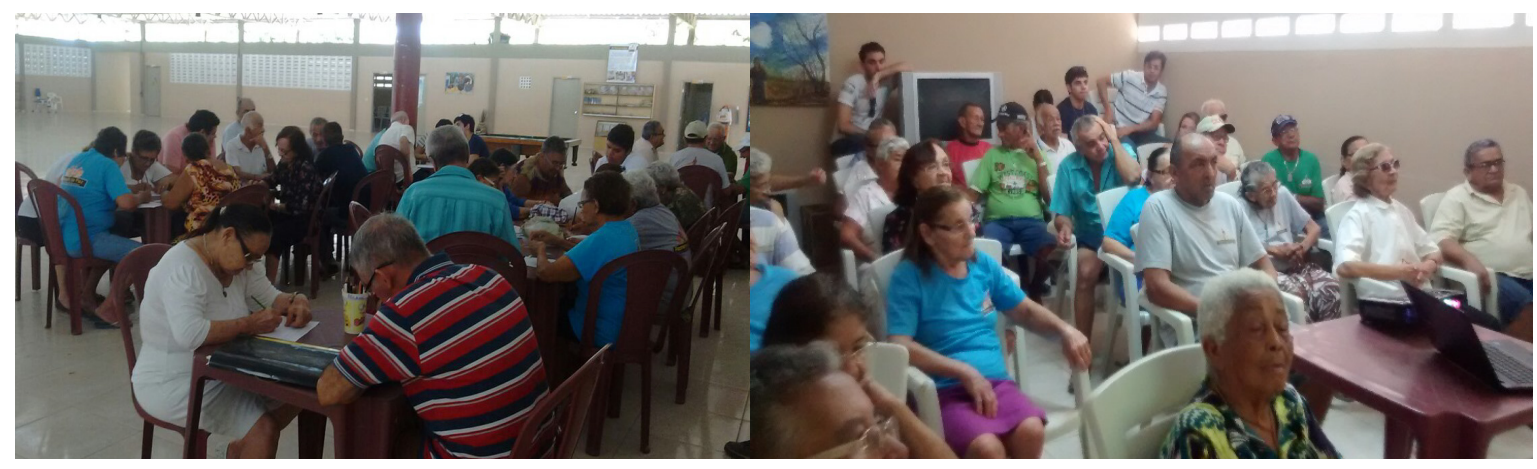

Figura 02 - Atividade educativa realizada através do projeto SAFI. Palestra "O uso racional de Medicamentos". 
No decorrer das atividades educativas, os acadêmicos realizavam conversas individuais referentes à terapia medicamentosa dos idosos, com orientação da posologia correta e a verificação de interações medicamentosas com alimentação e/ou com outros medicamentos, além de reações adversas que pudessem ocorrer pelo uso comitente de diversos medicamentos, evidenciando a prática da polimedicação e a ocorrência de efeitos colaterais. Para orientações sobre o uso como horários e a quantidade de medicamentos, foi disponibilizada aos idosos, uma tabela (Figura 03) com o objetivo de facilitar a utilização e minimizar efeitos tóxicos ocasionados pelo uso inadequado daqueles idosos que são polimedicados.

Observou-se que os idosos absorviam as inúmeras informações sobre os diversos assuntos tratados, ficando isso bastante explícito em algumas frases citadas pelos participantes: "Vocês deixaram o medicamento mais fácil", "Vocês foram diretamente ao assunto, com isso nós aprendemos melhor", "Antes eu tomava o chá e o remédio, aí vocês disseram o seguinte, qualquer remédio toma só com água", além da participação efetiva e mútua de dúvidas que surgiam sobre os assuntos.

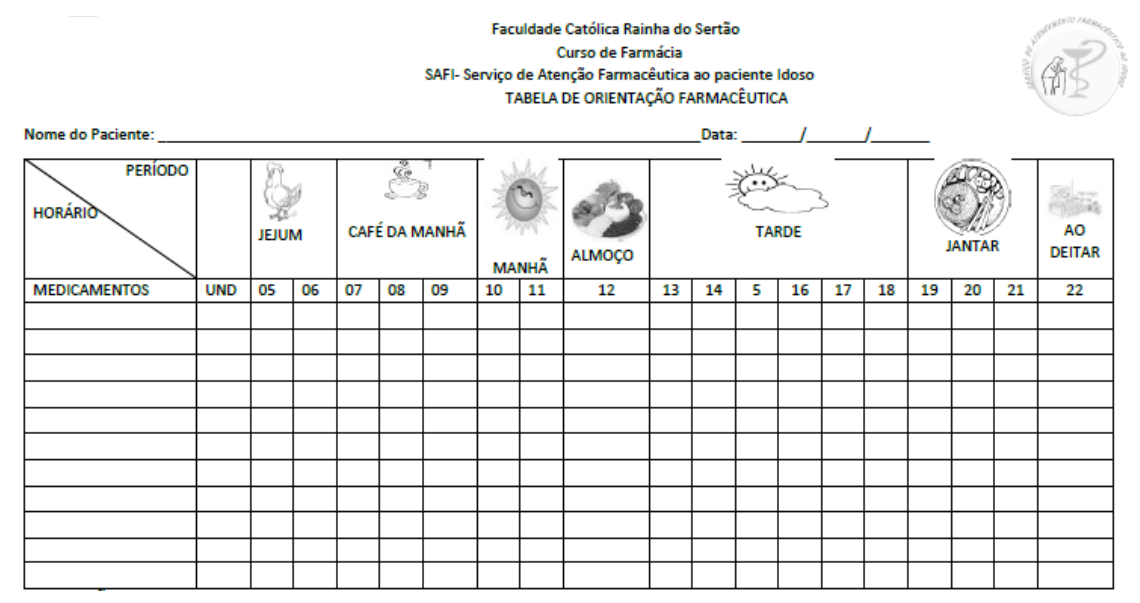

Figura 03 - Tabela de Orientação Farmacêutica.

Além disso, ações como aferição de pressão arterial e teste de glicemia (Figura 04) foram objetivadas para a melhor assistência da terapia dos idosos, com o monitoramento e orientação quanto aos resultados dos testes elevados ou minimizados tendo supervisão da Farmacêutica Maria Luísa Bezerra de Macedo Arraes (Registro do Conselho Regional de Farmácia:4557). De acordo com Brum et al (2012) ${ }^{15}$, exames de triagem mostram-se indispensáveis para o acompanhamento dos valores séricos de idosos hipertensos e diabéticos que para evitar alterações, e ainda avaliar a adesão da terapia medicamentosa.

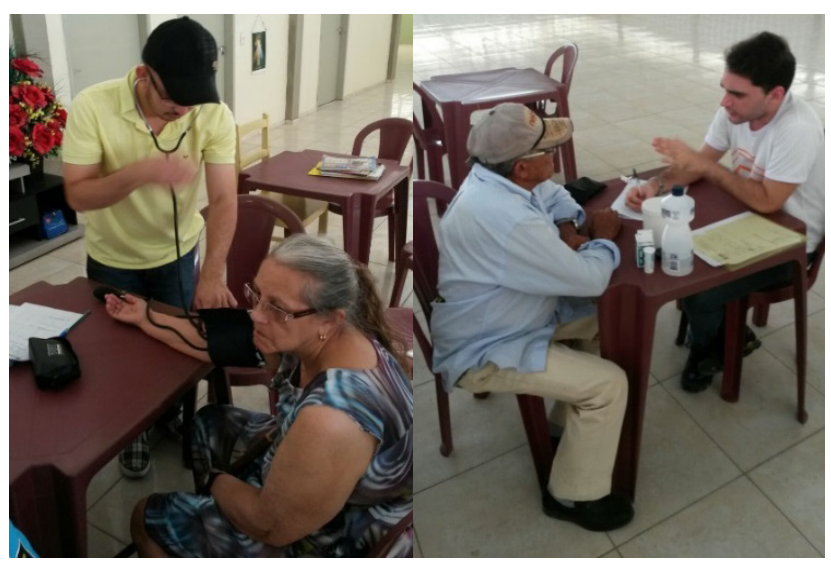

Figura 04 - Teste de glicemia capilar e verificação de Pressão arterial pelos acadêmicos de farmácia aos idosos da casa de apoio "Remanso da Paz". 
Ao final do projeto, foi disponibilizado um material educativo (Figura 06) sobre o uso racional de medicamentos aos idosos, com o propósito de fixar os assuntos e conhecimentos repassados, bem como favorecer a transmissão de informações para as demais pessoas envolvidas na saúde do idoso, como familiares e vizinhos. Pois de acordo com Gutierrez \& Minayo (2010) ${ }^{17}$, o cuidado com a saúde é realizado com uma ligação estável de uma série de elementos envolvidos, como estado emocional, físico, vínculo familiar, ambiente de trabalho, e residencial.

O informativo é composto por instruções para manter uma farmacoterapia eficaz, como evitar bebidas alcoólicas, realizar sempre o tratamento de acordo com a prescrição do profissional habilitado guardar os medicamentos em locais arejados e longe de crianças, não tomar medicamento vencido, tomar sempre os medicamentos na hora correta, evitando esquecer colocando-o em locais de fácil lembrete, ou usar despertadores.
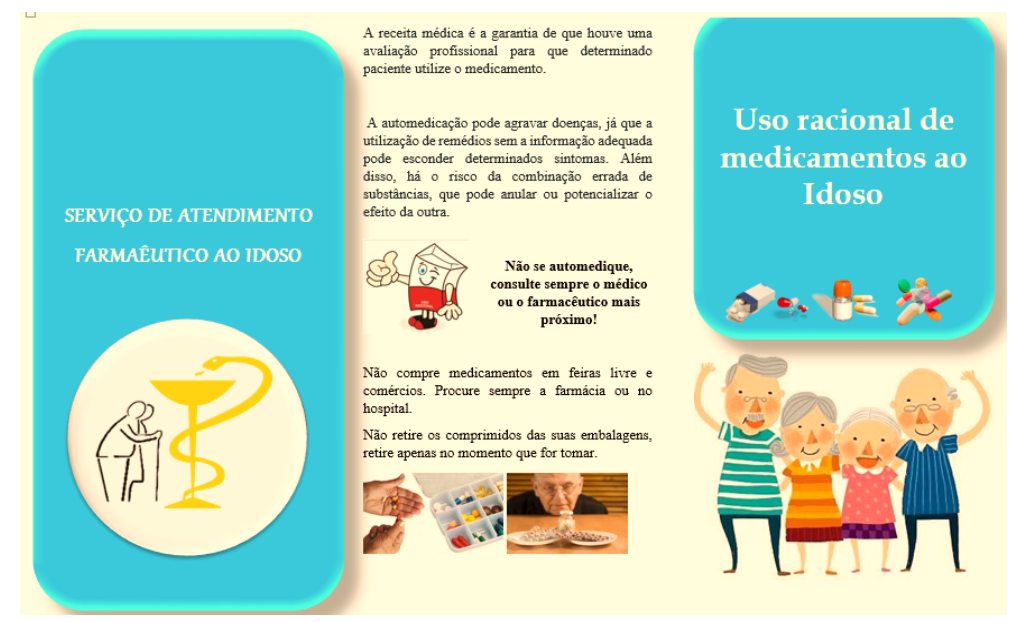

Figura 05 - Material Informativo sobre o uso racional de medicamentos aos idosos.

\section{Considerações Finais}

A prática da Atenção Farmacêutica está baseada na interação entre pacientes, familiares, profissionais de saúde e outros farmacêuticos. Esta interação social tem sido vista como a maior indutora da satisfação do paciente em relação aos serviços de saúde e ao desenvolvimento de um tratamento medicamentoso eficaz.

O projeto SAFI fortaleceu a adesão da farmacoterapia aos idosos, possibilitando a transmissão de informações sobre as doenças e o uso racional de medicamentos. Somado a isso, observa a contribuição à formação acadêmica dos estudantes de farmácia, que puderam expor seu conhecimento na prática diária, prestando a orientação Farmacêutica aos idosos, desenvolvendo uma terapia medicamentosa eficaz.

Evidencia-se, assim a importância da realização de atividades de educação em saúde à população idosa, e que no ato de cuidar do ser idoso e de seu cuidador não devemos focar nossas ações nos agravos de saúde , mas priorizar a promoção, manutenção e recuperação da saúde. Com isso, acredita-se que ao valorizar as trocas interpessoais permeadas pelo diálogo, ressaltando a importância do conhecimento popular poderemos superar as lacunas existentes nas práticas educativas de saúde tradicionais, e favorecer uma assistência farmacêutica qualificada.

\section{Referências}

1. Nóbrega O T, Karnikowski M G O. A terapia medicamentosa no idoso: cuidados na medicação. Ciên Saúde Coletiva [online]. 2005;10(2):309-13.

2. Aguiar P M, Lyra J D P, Silva D T, Marques T C. Avaliação da farmacoterapia de idosos residentes em instituições asilares no nordeste do Brasil. Lat. Am J Pharm. 2008;27(3):454-9.

3. Novaes M R C G. Assistência farmacêutica ao idoso - uma abordagem multiprofissional. Brasília: Thesaurus; 2007, p. 245.

4. Meneses A L L, Sá M L B. Atenção farmacêutica ao idoso: fundamentos e propostas. Geriatria \& Gerontologia. 2010;4(3):154-161. 
5. Almeida R O, Paiva C E Q, Ferreira S C H, Paiva Y C S. Prado. R. M. S. A importância da atenção farmacêutica para os pacientes geriátricos, 2014.

6. Costa R M, Lima V A B, Paiva I G, Sousa P T P, Lima L G. Uso de medicamentos por idosos: algumas considerações Geriatria \& Gerontologia. 2008; 3(2):126-131.

7. Silva A L. Estudo de utilização de medicamentos por idosos brasileiros. Belo Horizonte, 2009.

8. Silva et al. Polifarmácia em geriatria. Revista da AMRIGS, Porto Alegre, 56 (2): 164-174, abr.-jun. 2012.

9. Opas- Organização Pan-Americana da Saúde/Organização Mundial da Saúde. Assistência farmacêutica para gerentes municipais. / Organizado por MARIN, et al. Rio de Janeiro. 373p. 2002.

10. Gomes H O, Caldas C P. Uso Inapropriado de Medicamentos pelo Idoso: Polifarmácia e seus Efeitos. Revista do Hospital Universitário Pedro Ernesto, UERJ Ano 7, Janeiro / Junho de 2008.

11. Silva D C T, Silva K, Leal M J S. Educação na saúde do idoso. Faculdade Santa Marcelina. Anais. revista Kairós, São Paulo, 12(1), jan. 2009, pp. 275-330.

12. Chrispino M L, Andrade M. A educação em saúde aos idosos atendidos pelo psf. Informe-se em promoção da saúde, v.6, n.1.p.07-09, 2010.

13. Martins J J, Barra D C C, Santos T M, Hinkel V, Nascimento E R P, Albuquerque G L, Erdmann A L. Educação em saúde como suporte para a qualidade de vida de grupos da terceira idade Revista Eletrônica de Enfermagem, v. 09, n. 02, p. 443 - 456, 2007.

14. Brum D J T, Soccol K L S, Terra M G. Educação em saúde com idosos em grupos da "terceira idade". 2012.

15. Christiane Leite-Cavalcanti1, Maria da Conceição Rodrigues-Gonçalves1 , Luiza Sonia Rios-Asciutti1 e Alessandro Leite-Cavalcanti. Prevalência de doenças crônicas e estado nutricional em um grupo de idosos brasileiros. Rev. salud pública. 11 (6): 865-877, 2009.

16. Santos VCF, Kalsing A, Ruiz ENF, Roese A, Gerhardt TE. Perfil das inter- nações por doenças crônicas nãotransmissíveis sensíveis à atenção primária em idosos da Metade Sul do RS. Rev Gaúcha Enferm. 2013;34(3):124-131. 17. Gutierrez D M D, Minayo M C S. Produção de conhecimento sobre cuidados da saúde no âmbito da família. Ciência \& Saúde Coletiva, 15(Supl. 1):1497-1508, 2010.

\section{Sandna Larissa Freitas dos Santos}

Endereço para correspondência - Av. Plácida Castelo, n 1909,

Bairro: Centro, CEP: 63.900-000, Quixadá, CE, Brasil

E-mail: sandy.lary@hotmail.com.

Lattes: http://lattes.cnpq.br/7135170516978521.

Rainne de Oliveira Almeida - rainnealmeida26@hotmail.com

Carlos Eduardo Quirino Paiva - dudu-paiva1@hotmail.com

Karla Bruna Nogueira Torres Barros - karlabruna1@hotmail.com

Maria Luísa Bezerra de Macedo Arraes - mariaarraes@fcrs.edu.br

\section{Enviado em 21 de março de 2016. Aceito em 31 de maio de 2016.}

